\title{
EDITORIALS
}

\section{Colorectal Cancer Screening in the Elderly: The Need for Informed Decision Making}

\author{
Richard M. Hoffman, MD, MPH and Louise C. Walter, $M D^{2}$ \\ ${ }^{1}$ New Mexico VA Health Care System, University of New Mexico, Albuquerque, NM, USA; ${ }^{2}$ San Francisco VA Medical Center, University of \\ California, San Francisco, CA, USA.
}

$\mathrm{J}$ Gen Intern Med 24(12):1336-7

DOI: $10.1007 / \mathrm{s} 11606-009-1121-7$

(c) Society of General Internal Medicine 2009

$\mathrm{C}$ olorectal cancer screening works! We have convincing evidence from randomized trials of fecal occult blood tests (FOBT) showing that colorectal cancer screening reduces disease-specific morbidity and mortality ${ }^{1}$. Case-control studies suggest that endoscopy is also effective $\mathrm{e}^{2-4}$. Nonetheless, only about $60 \%$ of eligible adults are currently screened for colorectal cancer ${ }^{5}$. In contrast, nearly $75 \%$ of women are currently screened for breast cancer ${ }^{6}$, which has a similar level of evidence for efficacy as colorectal screening. More than $70 \%$ of men are currently screened for prostate cancer, which has uncertain efficacy ${ }^{7}$. The discordance between evidence and uptake of colorectal cancer screening has sparked considerable efforts to increase screening rates. However, the report by Kahi and colleagues suggests that these efforts may have the unintended consequence of promoting screening recommendations that fail to adequately consider the risks and benefits of screening in the context of a patient's health status and life expectancy ${ }^{8}$.

Perhaps cancer screening is like the forward pass in football. Darrell Royal the legendary coach at the University of Texas has said, "When you throw a pass, only three things can happen, and two of "em are bad." Colorectal cancer screening can reduce morbidity and mortality, the supreme accomplishment of a screening program. However, the absolute benefit to a screened population is small; most people will not benefit because they do not have early-stage colorectal cancer or polyps destined to become malignant. The Minnesota Colon Cancer Control Study showed that annual FOBT screening reduced colorectal cancer mortality by about 3 deaths per 1000 people screened after 13 years of follow up ${ }^{9}$. Similarly, screening reduced the risk for cancer diagnosis by just 7 per 1,000 people screened after 18 years of follow up ${ }^{10}$. This small likelihood of future benefit must then be weighed against the more immediate potential downsides of screening, including false positive tests, the costs and risks of invasive diagnostic tests, treatment complications, and the risk for overdiagnosing cancer-finding disease that is not destined to cause any problems during a patient's lifetime.

The latter problem is particularly pertinent for cancer screening in sick and elderly patients. To minimize inappropriate and potentially harmful screening, the US Preventive Services Task Force has recently provided explicit guidelines-

Published online October 2, 2009 no screening after age 85 and personalized screening decisions for those ages 76 to $85^{11}$. These recommendations are partly based on the trial data showing that colorectal cancer survival curves did not diverge until 5 years after the start of screening. This interval reflects that it often takes many years between when a cancer could be first detectable by screening until when it would have grown large enough to cause symptoms or death. The recommendation for "personalized" screening decisions really is a call for informed decision making. Older patients, especially those who have serious medical illnesses, need to be aware that they are least likely to live long enough to receive any benefit from cancer screening and more likely to experience the complications from tests and treatments.

The study by Kahi and colleagues did not address whether physicians would elicit patient preferences or support informed decision-making, but the fact that respondents reported that they would offer screening to patients over age 85 or to 75 -year-old patients with cancers or severe heart failure is disconcerting. These patients clearly are very unlikely to derive any benefit from screening and are at substantial risk for being harmed. While these are survey data and respondents were presented with hypothetical scenarios, it appears that actual screening practices may indeed be quite inappropriate. A recent cohort study of older veterans at four VA medical centers used linked VA and Medicare data to describe screening practices ${ }^{12}$. During calendar years 2001 and 2002, $37.6 \%$ of veterans aged 80 and older who were due for colorectal cancer screening underwent screening with FOBT, endoscopy, or barium enema. Strikingly, among this group, the screening rate was $34 \%$ for those with severe comorbidity (defined by Charlson-Deyo scores $\geq 4$ ), only slightly lower than the $38 \%$ screening rate for those who had comorbidity scores of 0 .

Why is this happening? Several authors have suggested that organizational zeal to boost screening rates by converting practice guidelines into performance measures can increase the rate of inappropriate screening in the sick and elderly ${ }^{13,14}$. Similarly, public service messages targeting the public may oversimplify the screening message- “just do it"-rather than presenting accurate, balanced information about the pros and cons of screening. Such oversimplified messages likely contribute to why many patients do not make informed decisions about cancer screening and why frail elderly patients continue to undergo cancer screening, even when the risks are substantial and the benefits are remote.

What can be done about this? Clearly, organizations need to more carefully balance performance measures and financial incentives with evidence-based guidelines. Quality-of-care 
assessments must identify and reward efforts that target patients most likely to benefit from screening rather than those efforts that merely increase screening rates. Optimizing the targeting of cancer screening also may require changes in how preventive care is delivered. The current system for delivering preventive care relies on finding opportunities for screening when patients see clinicians for medical problems, which targets screening to elderly patients with more serious comorbid conditions because they see clinicians more frequently. This system misses healthy patients who see clinicians less frequently. In addition, both patients and clinicians need to be more aware of the potential risks associated with screening, especially for persons with advanced age and serious comorbidities. When two out of three outcomes for the forward pass are bad, the coach and players need to carefully study the playbook and make an informed decision.

Corresponding Author: Richard M. Hoffman, MD, MPH; New Mexico VA Health Care System, University of New Mexico, 1501 San Pedro Drive SE, Albuquerque, NM 87108, USA (email: rhoffman@unm.edu).

\section{REFERENCES}

1. Hewitson P, Glasziou P, Watson E, Towler B, Irwig L. Cochrane systematic review of colorectal cancer screening using the fecal occult blood test (hemoccult): an update. Am J Gastroenterol. 2008;103 (6): 1541-9.
2. Selby JV, Friedman GD, Quesenberry CP Jr., Weiss NS. A case-control study of screening sigmoidoscopy and mortality from colorectal cancer. N Engl J Med. 1992;326(10):653-7.

3. Muller AD, Sonnenberg A. Protection by endoscopy against death from colorectal cancer. A case-control study among veterans. Arch Intern Med. 1995;155(16):1741-8.

4. Baxter NN, Goldwasser MA, Paszat LF, Saskin R, Urbach DR, Rabeneck L. Association of colonoscopy and death from colorectal cancer. Ann Intern Med. 2009;150(1):1-8.

5. Use of colorectal cancer tests-United States, 2002, 2004, and 2006. MMWR Morb Mortal Wkly Rep. 2008;57(10):253-8.

6. Use of mammograms among women aged $>$ or $=40$ years-United States, 2000-2005. MMWR Morb Mortal Wkly Rep. 2007;56(3):49-51.

7. Sirovich BE, Schwartz LM, Woloshin S. Screening men for prostate and colorectal cancer in the United States: does practice reflect the evidence? Jama. 2003;289(11):1414-20.

8. Kahi CJ, van Ryn M, Juliar B, Stuart JS, Imperiale T. Provider recommendations for colorectal cancer screening in elderly veterans. J Gen Intern Med. 2009. doi:10.1007/s11606-009-1110-x

9. Mandel JS, Bond JH, Church TR, et al. Reducing mortality from colorectal cancer by screening for fecal occult blood. Minnesota Colon Cancer Control Study. N Engl J Med. 1993;328(19):1365-71.

10. Mandel JS, Church TR, Bond JH, et al. The effect of fecal occult-blood screening on the incidence of colorectal cancer. N Engl J Med. 2000;343 (22): 1603-7.

11. Screening for colorectal cancer: U.S. Preventive Services Task Force recommendation statement. Ann Intern Med. 2008;149(9):627-37.

12. Walter LC, Lindquist $\mathbf{K}$, Nugent $\mathbf{S}$, et al. Impact of age and comorbidity on colorectal cancer screening among older veterans. Ann Intern Med. 2009; 150(7):465-73.

13. Walter LC, Davidowitz NP, Heineken PA, Covinsky KE. Pitfalls of converting practice guidelines into quality measures: lessons learned from a VA performance measure. Jama. 2004;291(20):2466-70.

14. Fisher DA, Galanko J, Dudley TK, Shaheen NJ. Impact of comorbidity on colorectal cancer screening in the veterans healthcare system. Clin Gastroenterol Hepatol. 2007;5(8):991-6. 